\title{
Fourier Analysis to Measure Diffusion Coefficients and Resolve Mixtures on a Continuous Electrophoresis Chip
}

\author{
A. Estévez-Torres, ${ }^{\dagger}$ C. Gosse,${ }^{\S, \star}$ T. Le Saux,${ }^{\dagger}$ J. - F. Allemand,${ }^{\sharp}$ \\ V. Croquette, ${ }^{\sharp}$ H. Berthoumieux,${ }^{\dagger}$ A. Lemarchand, ${ }^{\ddagger}$ L. Jullien ${ }^{\dagger, \star}$ \\ ${ }^{\dagger}$ Ecole Normale Supérieure, \\ Département de Chimie, UMR CNRS ENS Université Paris6 8640, \\ 24, rue Lhomond, 75005 Paris, France. \\ E-mail: Ludovic.Jullien@ens.fr \\ $\S$ Laboratoire de Photonique et de Nanostructures, LPN-CNRS, \\ Route de Nozay, 91460 Marcoussis, France. \\ E-mail: charlie.gosse@lpn.cnrs.fr \\ ${ }^{\sharp}$ Ecole Normale Supérieure, \\ Département de Physique, UMR CNRS ENS Université Paris6 and Paris7 8550, \\ 24, rue Lhomond, 75005 Paris, France. \\ $\ddagger$ Université Pierre et Marie Curie Paris 6 , \\ Laboratoire de Physique Théorique de la Matière Condensée, UMR CNRS 7600, \\ 4, place Jussieu, 75252 Paris Cedex 05, France.
}

\section{Supporting Information}

- Theoretical derivation of the expression of the Fourier transform of the concentration $F(x, y)$ with respect to $y$;

- PCR protocol for syntheses of the dsDNA fragments;

- Detailed microfabrication protocol for the bottom PDMS layer;

- Finite Element Method simulations;

- Measurements of diffusion coefficients in time-based experiments;

- Correction of the optical pattern in the Fourier space;

- Validation of the procedure for data processing;

- Supplementary Tables (Tables S-2 and S-3);

- Supplementary Figures (Figures S-6-S-10). 


\section{Theoretical derivation of the expression of the Fourier transform of the concentration $F(x, y)$ with respect to $y$}

We here detail the calculation of the migration-diffusion pattern in the Fourier space for the boundary conditions reported in the Main Text.

The integration constants $C_{ \pm}$in eq 3 are deduced from the boundary conditions: $F(0, y)$ at $x=0$ and $F(a, y)$ at $x=a$. We find:

$$
\begin{aligned}
& C_{-}=\frac{\exp \left(\lambda_{+} a\right) \tilde{F}(0, q)-\tilde{F}(a, q)}{\exp \left(\lambda_{+} a\right)-\exp \left(\lambda_{-} a\right)} \\
& C_{+}=\frac{\tilde{F}(a, q)-\exp \left(\lambda_{-} a\right) \tilde{F}(0, q)}{\exp \left(\lambda_{+} a\right)-\exp \left(\lambda_{-} a\right)}
\end{aligned}
$$

In the experimental conditions considered, $\lambda_{+} a \gg 1$. Indeed $\lambda_{+} a \geq v_{x} a / 2 D$ from eq 4 and typical values for $v_{x}, D$ and $a$ are here $10^{-5} \mathrm{~ms}^{-1}, 10^{-10} \mathrm{~m}^{2} \mathrm{~s}^{-1}$ and $10^{-2} \mathrm{~m}$ respectively: $\lambda_{+} a \geq 5 \times 10^{2}$. Taking into account that $\lambda_{-} a \leq 0$, we write: $\exp \left(\lambda_{+} a\right)-\exp \left(\lambda_{-} a\right) \simeq \exp \left(\lambda_{+} a\right), C_{-} \simeq \tilde{F}(0, q)-$ $\tilde{F}(a, q) \exp \left(-\lambda_{+} a\right)$ and $C_{+} \simeq \exp \left(-\lambda_{+} a\right) \tilde{F}(a, q)-\exp \left[\left(\lambda_{-}-\lambda_{+}\right) a\right] \tilde{F}(0, q)$. Eq 3 of the Main Text now provides:

$$
\tilde{F}(x, q)=\tilde{F}(0, q)\left\{\exp \left(\lambda_{-} x\right)-\exp \left[\left(\lambda_{-} a+\lambda_{+}(x-a)\right]\right\}+\tilde{F}(a, q) \exp \left[\lambda_{+}(x-a)\right]\right.
$$

Taking into account that $\left(\lambda_{+}-\lambda_{-}\right) a>\lambda_{+} a \gg 1$, we consequently derive eq 5 of the Main Text for small values of the abscissa, $x \ll a$. 


\section{PCR Protocol for Syntheses of the dsDNA Fragments}

Double-stranded DNA was synthesized by PCR using $\lambda$-DNA (Sigma-Aldrich, St-Louis, MO) as a template. Three sets of primers (L1-R1, L4-R2, and L1-R2; HPLC grade from IBA, Göttingen, Germany; see Table S-1 for sequences) were selected with the Primer3 software ${ }^{1}$ to obtain products of three different sizes (151, 438, and 1257bp, respectively). The reaction was carried out with the Expand High Fidelity PCR System (Roche Diagnostics, Meylan, France) following the manufacturer instructions. The products were subsequently purified by exclusion on either Chroma Spin TE-100 columns (BD Biosciences, Palo Alto, CA) for the 151bp and 438bp fragments or Chroma Spin TE-400 for the 1257bp ones. Finally, the samples were concentrated by ethanol precipitation, dissolved in $25 / 50 \mathrm{mM} \mathrm{NaOH} / \mathrm{Hepes}$ pH 7.5 buffer, and analyzed by electrophoresis on a $1 \%$ agarose gel. After quantification by UV spectrophotometry, the PCR yield could be estimated between 16 to 48 ng of DNA per $\mu \mathrm{L}$ of initial PCR mix.

Table S-1. Names and sequences (5'-3' orientation) of the PCR primers used in this study.

\begin{tabular}{lr}
\hline Name & Sequence \\
\hline & \\
L1 & GACGATTCATAAGTTCCGCTGT \\
R1 & GTTACGCCACCAGTCATCCT \\
L4 & AAAGCCAGAACTCCCCGTAT \\
R2 & GGTATTCCCCTCAGTGTCCAG \\
\hline
\end{tabular}




\section{Detailed Microfabrication Protocol for the Bottom PDMS Layer}

The device is composed of a circular glass cover-slip $(0.17 \mathrm{~mm}$ thick, $40 \mathrm{~mm}$ diameter; Menzel-Gläser, Germany) and two $\sim 3 \mathrm{~mm}$ thick cross-linked poly(dimethylsiloxane) (PDMS) layers (Figure S-1). The elastomeric bottom stamp, in contact with the glass, contains the thin analysis chamber connected to six reservoirs through arrays of microchannels. On top of it, a second PDMS stamp is used to shape four macroreservoirs accommodating four electrodes.

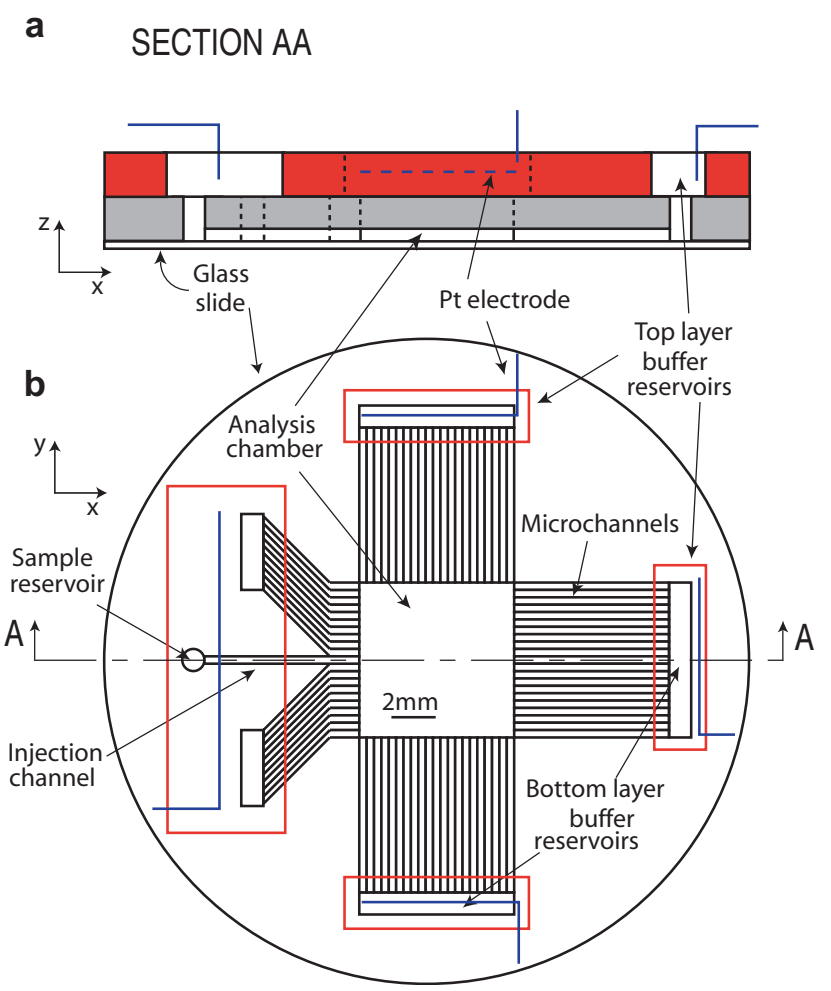

Figure S-1. Cross-section and top views of the two-layer electrophoresis device.

The bottom layer fabrication is decomposed in two steps. First, a positive PDMS master including the microfluidics channels and the reservoirs is obtained by classical soft-lithography protocols. Then, by counter-molding, it yields a negative epoxy master which is used for routine production of the PDMS bottom layer.

The initial PDMS master is obtained as follows: (i) The layout, designed with the L-edit CAD program (Tanner EDA, Monrovia, CA), is printed on a transparency at 25400 dpi resolution (Selba, Versoix, Switzerland); (ii) Features are transferred into a $10 \mu \mathrm{m}$ thick photoresist layer by optical lithography : AZ9260 (AZ Electronic Materials, Wiesbaden, Germany) is spin-coated on a 2" silicon wafer (IEMT, Warsaw,Poland) pretreated with Microposit Primer (Rohm and Haas Electronic Materials, Coventry, UK), 5 min insolation is then performed on a MJB3 aligner (i-line; SUSS MicroTec, Garching, Germany), and channels are finally developed in AZ400K (Clariant, Frankfurt, Germany); (iii) The processed Si wafer is pretreated with trimethylchlorosilane (TMCS; Acros Organics, Geel, Belgium) vapor for $5 \mathrm{~min}$; (iv) PDMS base and curing agent (Bayer, Leverkusen, Germany) are mixed at a 10:1 (w/w) 
ratio, poured onto the AZ9260 photoresist master, and degassed at 50 mbar; (v) After baking at $60^{\circ} \mathrm{C}$ for $2 \mathrm{~h}$, the polymerized replica is peeled off and the reservoirs are cut out under a stereomicroscope to yield the PDMS master mould.

In a second step, the epoxy master is fabricated as follows: epoxy crystal resin (Gedeo, Gemenos, France) is mixed following manufacturer instructions, poured onto the PDMS master mould, degassed, cured for $2-3$ days at room temperature and eventually peeled off.

Then, to repeatedly fabricate PDMS bottom layer, the epoxy master mould is treated with TMCS vapor, filled with the PDMS mix, degassed and cured for $2 \mathrm{~h}$ at $60^{\circ} \mathrm{C}$. 


\section{Finite Element Method Simulations}

The profiles of electric potential and concentration are computed with the Finite Element Method software Comsol Multiphysics (Comsol, Stockholm, Sweden) using a simulation cell of length $\ell_{x}$ and width $\ell_{y}$.

\section{Electric Potential Computation}

\section{Laplace Equation}

The electric field $\vec{E}$ is computed using the Gauss equation:

$$
\operatorname{div}(\vec{E})=\frac{\rho}{\epsilon_{r} \epsilon_{0}}
$$

where $\rho$ is the local charge density and $\epsilon_{r} \epsilon_{0}$ the medium permittivity. $\rho=0$ results from modelling the buffer as an isotropic and isothermal conductor where electroneutrality is verified at any point. Then the definition $\vec{E}=-g \overrightarrow{r a} d V$ for the electric potential $V$ yields the Laplace equation that is solved by Comsol Multiphysics:

$$
\Delta V=0
$$

\section{Boundary Conditions for the Electric Field}

- The PDMS is considered to be totally insulating. Thus a zero normal current boundary condition is set at its surface:

$$
\vec{J} . \vec{n}=0
$$

where $\vec{J}$ is the current density vector and $\vec{n}$ the unit vector normal to any surface (Figure S-2a).

- The lateral boundaries of the simulation cell, parallel to the $x$-axis, are also set as insulating. Indeed, considering the chamber as an infinite arrays of channels, periodicity imposes $E_{y}\left(x, \ell_{y} / 2\right)=$ $E_{y}\left(x,-\ell_{y} / 2\right)$ whereas symmetry with respect to the $x$-axis yields $E_{y}\left(x, \ell_{y} / 2\right)=-E_{y}\left(x,-\ell_{y} / 2\right)$, hence resulting in $E_{y}\left(x, \ell_{y} / 2\right)=E_{y}\left(x,-\ell_{y} / 2\right)=0$.

- We impose fixed voltage boundary conditions at both channels entries and chamber exit.

\section{Concentration Profile Computation}

\section{Migration-Diffusion Equation}

The equation to be solved by the Finite Element Method software can easily be derived from the equation of matter conservation:

$$
\operatorname{div}\left(\overrightarrow{J_{F}}\right)=\frac{\partial F}{\partial t}
$$

where $F$ is the analyte concentration and $\overrightarrow{J_{F}}$ its flux density. Taking into account both diffusion and electrophoretic migration $\left(\overrightarrow{J_{F}}=-D g r \vec{a} d F+F . \mu \vec{E}\right)$ under stationary conditions (i.e. with $\partial F / \partial t=0$ ), it yields the classical migration-diffusion equation :

$$
\operatorname{div}(-D g r \vec{a} d F+F \cdot \mu \vec{E})=0 .
$$




\section{Boundary Conditions for the Analyte}

- We adopt a constant concentration at the entrance of the injection channel as the boundary condition and we set $F=F_{0}$ (Figure S-2b).

- Concerning the chamber exit, we consider that the analyte peak broadening along the $x$-axis results in a smooth concentration gradient at the chamber exit, leading to neglect the diffusive flux in front of the analyte transport by electrophoresis. Thus we selected the zero diffusive flux condition in Comsol Multiphysics :

$$
-D g r a d F \cdot \overrightarrow{u_{x}}=0
$$

that was validated a posteriori.

- The PDMS is impermeable; A boundary condition of zero normal flux is set at its surface:

$$
\overrightarrow{J_{F}} \cdot \vec{n}=0
$$

- Eventually, we impose a null solute concentration at the lateral edges of the simulation cell, i.e. parallel to the $x$-axis, and at all the remaining channels entries. It is a priori justified by the fact that those areas are very far from the migration-diffusion parabola-like pattern.

\section{Results and Hypothesis Evaluation}

The potential values at both edges of the simulation cell were calculated to yield a $E_{x}=8 \times 10^{3} \mathrm{Vm}^{-1}$ electric field inside the analysis chamber. For computation, we used the diffusion coefficient $D$ and apparent electrophoretic mobility $\mu$ of fluorescein that we measured on chip : $340 \times 10^{-12} \mathrm{~m}^{2} \mathrm{~s}^{-1}$ and $18.1 \times 10^{-9} \mathrm{~m}^{2} \mathrm{~V}^{-1} \mathrm{~s}^{-1}$, respectively.

\section{Size of the Simulation Cell}

The characteristic lengths $\ell_{x}$ and $\ell_{y}$ have been chosen to minimize artifacts from the boundary effects. Typical experimental images have a $1024 \times 1024$ pixels size, which corresponds, on chip, to a $875.50 \mu \mathrm{m}$ side square. Thus we chose a simulation cell of width $\ell_{y}=880 \mu \mathrm{m}$ and adopted a cell length $\ell_{x}=3 \ell_{y}{ }^{1}{ }^{1}$ We additionally located the chamber entry such that the resistive channels represent one third of the total $x$-dimension.

\section{Electric Field Homogeneity}

Figure S-2c displays the results of the electric potential simulation. A $\ell_{0}=40 \mu \mathrm{m}$ inhomogeneity limit clearly appear when plotting the electric field $x$-component (Figure S-2e) around the chamber entry.

\footnotetext{
${ }^{1}$ A larger simulation area with $\ell_{y}=1280 \mu \mathrm{m}$ yields similar values of the electric potential and of the concentration (the discrepancy is smaller than $2 \%$; Figure S-3a and b) which underlines the absence of any artifact related to the finite size of the computation area.
} 


\section{Implementing the Appropriate Migration-Diffusion Equation in Comsol Multiphysics}

Eq 8 can be developed in:

$$
-D . \Delta F+\mu \cdot \operatorname{div}(F \cdot \vec{E})=0
$$

which yields:

$$
-D \cdot \Delta F+\mu[\vec{E} \cdot g r \overrightarrow{r a d} F+F \cdot \operatorname{div}(\vec{E})]=0 .
$$

Assuming the electroneutrality verified throughout the buffer, i.e. $\operatorname{div}(\vec{E})=0$ (eq 4), it finally writes:

$$
-D \cdot \Delta F+\mu \vec{E} \cdot g \overrightarrow{r a} d F=0 .
$$

From a mathematical point of view, eqs 8 and 13 are equivalent. However, we observed that the results of the simulations differ, depending on which implementation mode was selected in Comsol Multiphysics. Indeed, as the conservative option, corresponding to eq 9, yields acceptable results, the non-conservative option, corresponding to eq 13 , result in abnormal solute accumulation at the injection nozzle. Additionnally, in this last case, we noticed that the fluorescein concentration tends towards the one found by the conservative solver when the mesh was refined. Consequently, we hypothesized some technical origin for the discrepancy between the two computing strategies. It was further confirmed by plotting the electric field divergent : $\operatorname{div}(\vec{E})$ is not always null and can even reach non-negligeable values around angular edges of insulating materials. Consequently, eq 13 does not correspond anymore to eq 8 and the non-conservative solving option should be avoided to address this kind of coupled equations system.

\section{Validity of the Null Concentration Boundary Condition}

For computing the concentration profile, we impose a null solute concentration at the lateral boundaries of the simulation area. To validate this hypothesis, we calculated the normalized concentration in a larger cell and plotted the results along what would be the edge of the normal simulation cell (Figure S-3c). $F / F_{0}$ values below $0.01 \%$ confirm the absence of any analyte at this location and thus validate the present boundary condition.

\section{Validity of the Zero-Diffusive Flux Boundary Condition}

To check if Eq 9 is an appropriate boundary condition for simulations of concentration profiles, we applied the strategy used in the preceding subsection. The diffusive and the convective flux, calculated in a larger cell, are compared for positions corresponding to the normal cell edge (Figure S-3d). $D \partial C / \partial x$ always represents less than a few percents of the total analyte flux along $x$, hence validating the zero diffusive flux boundary condition implemented in Comsol Multiphysics for the chamber exit.

\section{Diffusion Coefficient Extraction by Fourier Transform Analysis}

Figure S-2d displays the simulated concentration profiles used for Fourier modes computation and subsequent $D_{n}$ extraction, resulting in Figure 3c of the Main Text. 
a

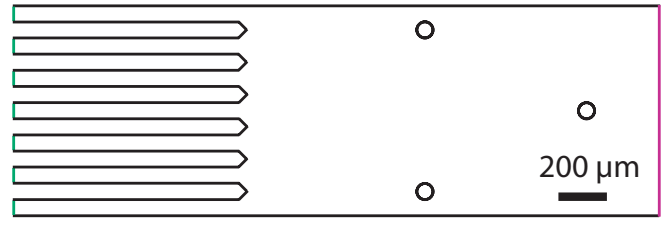

C

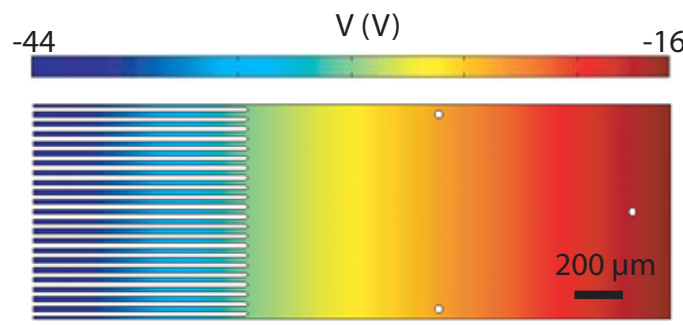

b

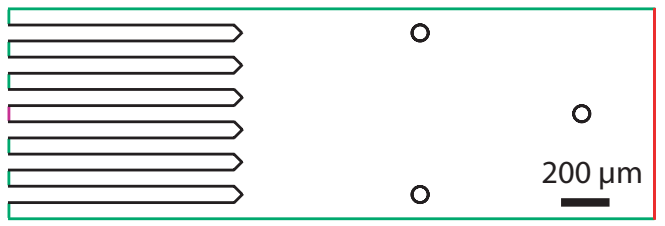

$\mathrm{d}_{0} \quad \mathrm{~F} / \mathrm{F}_{0}$

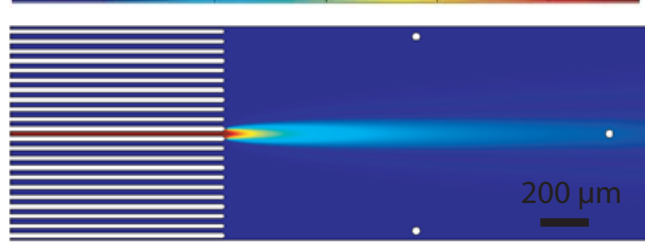

e

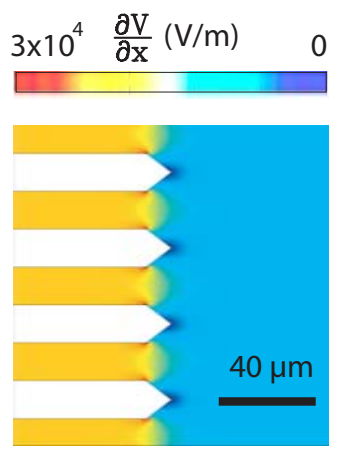

Figure S-2. Finite Element Method simulations of the experiment presented in the Figure 3 of the Main Text: Fluorescein at a concentration $F_{0}=5 \mu \mathrm{M}$ is injected under a $300 \mathrm{~V}$ potential drop in $\mathrm{NaOH} / \mathrm{Hepes} 25 / 50 \mathrm{mM}$ $\mathrm{pH} 7.5,0.1 \%$ (w/w) PDMA buffer at $20^{\circ} \mathrm{C}$. The simulation cell is $880 \mu \mathrm{m}$ wide and $2640 \mu \mathrm{m}$ long. (a) Boundary conditions for the electric field : (-) corresponds to electric insulation and (-) and (-) to imposed voltages, $-44 \mathrm{~V}$ and $-16 \mathrm{~V}$, respectively. These two last values were simply computed assuming that the resistance per unit length in the channels is double than the one in the chamber. To improve readability, the scheme is not to scale. (b) Boundary conditions for the analyte : (-) corresponds to a zero normal flux, $(-)$ to a zero diffusive flux, and (-) and (-) to imposed concentrations, 0 and $F_{0}$, respectively. The scheme is not to scale. (c) Values of the electric potential in the analysis chamber. (d) Migration-diffusion pattern for the solute (concentration is normalized by $F_{0}$ ). (e) Zoom on the $\partial V / \partial x$ inhomogeneities at the injection nozzle. 
a

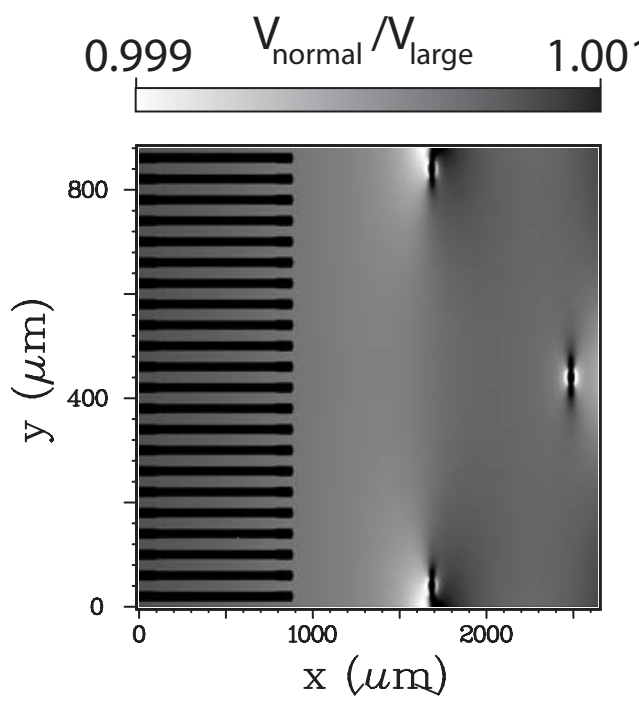

C

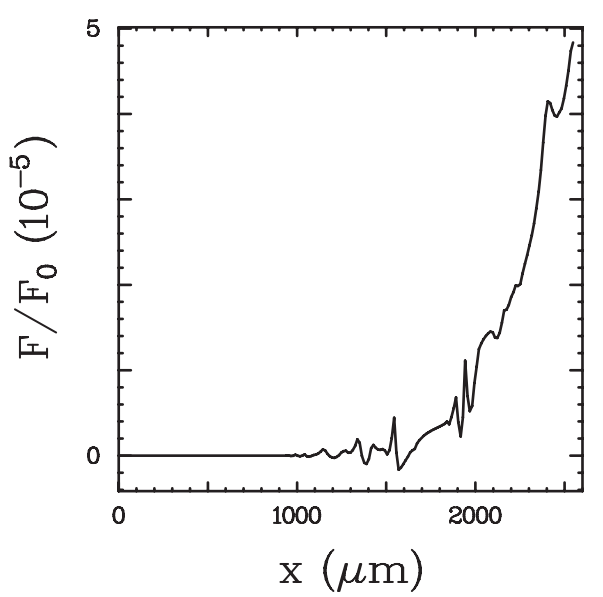

b

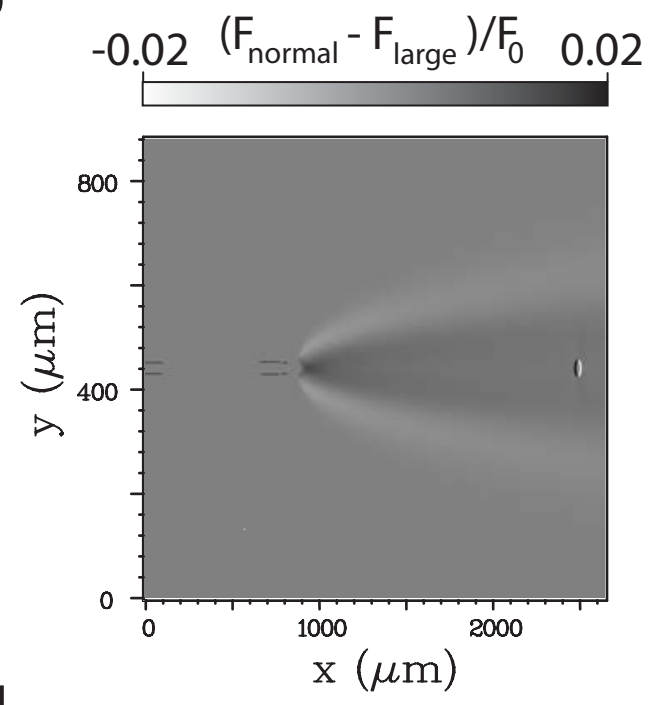

d

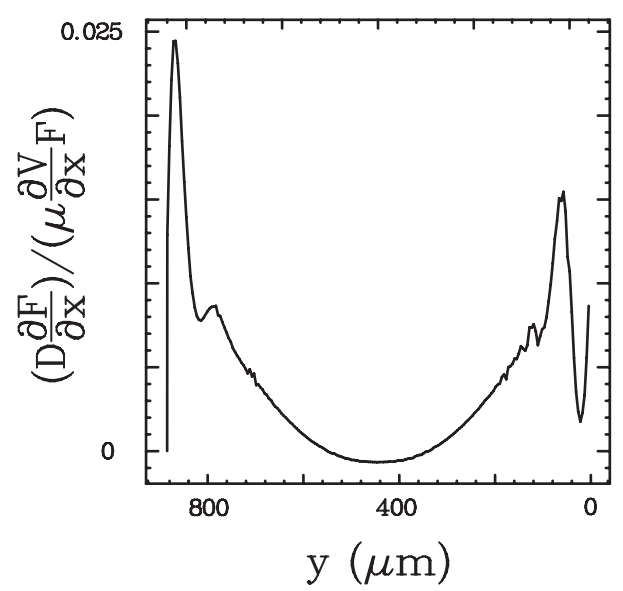

Figure S-3. Validation of the hypotheses underlying the boundary conditions used for Finite Element Model simulations. Computations are performed in a cell of either the normal size, $880 \mu \mathrm{m}$ wide and $2640 \mu \mathrm{m}$ long, or $\sim 1.45$ larger, $1280 \mu \mathrm{m}$ wide and $3840 \mu \mathrm{m}$ long. $5 \mu \mathrm{M}$ fluorescein is injected under a $300 \mathrm{~V}$ potential drop in $\mathrm{NaOH} / \mathrm{Hepes} 25 / 50 \mathrm{mM} \mathrm{pH} 7.5,0.1 \%$ (w/w) PDMA buffer at $20^{\circ} \mathrm{C}$. (a) Comparison between the electric potentials found for the two different simulation cell sizes. Values obtained in the larger cell were divided by the ones obtained in the normal one. (b) Comparison between the solute migration-diffusion patterns computed with the two different simulation cell sizes. Normalized values obtained in the normal cell were substracted to the ones obtained in the larger one. (c) Dependence along $x$ of the concentration $F$; values are calculated at $y=440 \mu \mathrm{m}$ in the large cell. (d) Dependence along $y$ of the ratio between the $x$-components of the diffusive and convective flux; values are calculated at $x=1760 \mu \mathrm{m}$ in the large cell. 


\section{Measurements of Diffusion Coefficients in Time-Based Experiments}

In this work, we did not only perform continuous stationary experiments as reported in the Main Text. We also designed and performed time-based experiments in which the spreading along the $y$-axis of an initial concentration profile generated along the $x$-axis was analyzed over time.

Two types of time-based experiments were carried out. In the zero-field time-based experiments, the initial condition along $x$ relaxes by diffusion in the absence of any electric field. In $E_{y}$-field time-based experiments, we additionally apply an alternative electric field along $y$ : Migration now superimposes to diffusion. The comparison between the diffusion coefficients measured in both experiments allows us to evaluate any possible electric field-induced dispersion that could superimpose to the Brownian motion in the present device.

\section{Theory}

Time-based experiments start by continuously applying an electric field $E_{x}$ along the $x$-axis to generate a stationary migration-diffusion pattern in the $\mathbf{F}$ species. After $E_{x}$ has been switched off, the evolution of a concentration profiles along $y$, averaged over a characteristic length $\Delta x(\sim 10 \mu \mathrm{m})$, is followed over time. With diffusion coefficients $D \sim 10^{-10}-10^{-12} \mathrm{~m}^{2} \mathrm{~s}^{-1}$ and velocity along $x v_{x} \sim 10^{-4} \mathrm{~ms}^{-1}$, $\frac{D}{v_{x}} q^{2} \Delta x \ll 1$ and thus $\tilde{F}(x, q)$ is constant at the $\Delta x$ length scale: Migration dominated diffusion along $x$ during the preparation of the initial condition. Therefore this initial concentration profile can be therefore considered as independent on $x$. Then the problem becomes unidimensional.

We consequently adopt eq 14 to model the subsequent relaxation of the preceding initial condition by diffusion-migration:

$$
\frac{\partial F(y, t)}{\partial t}=-v_{y} \frac{\partial F(y, t)}{\partial y}+D \frac{\partial^{2} F(y, t)}{\partial y^{2}}
$$

where $v_{y}$ designates the velocity of $\mathbf{F}$ along $y .^{2}$ Introducing the spatial Fourier transform along $y$, $\tilde{F}(q, t)=\frac{1}{\sqrt{2 \pi}} \int_{-\infty}^{\infty} F(y, t) e^{-i q y} d y$, we derive the solution of eq 14 in the Fourier space:

$$
\tilde{F}(q, t)=\tilde{F}(q, 0) \exp \left(-D q^{2} t\right) \exp (i q v t)
$$

where $\tilde{F}(q, 0)$ is the Fourier transform of the initial condition $F(y, 0)$. The amplitude of eq 15 :

$$
|\tilde{F}(q, t)|=|\tilde{F}(q, 0)| \exp \left(-D q^{2} t\right)
$$

shows that $D$ can be simply extracted from an exponential fit in zero-field and $E_{y}$-field time-based experiments.

\section{Data Acquisition}

We first generate the initial condition by applying a strong electric field $E_{x}$, several $\mathrm{kVm}^{-1}$, along the $x$-axis (Figure S-4a). At $t=0, E_{x}$ is switched off and the diffusion of the analyte along the $y$-axis is

\footnotetext{
${ }^{2} \mathrm{We}$ apply a squared time-periodic electric field yielding $v(t)=v_{y}$ (for $0<t \leq \mathrm{T} / 2$ ) and $v(t)=-v_{y}$ (for $\mathrm{T} / 2<t \leq \mathrm{T}$ ) in the $E_{y}$-field time-based experiment. Then $v_{y}$ remains only constant over half a period $T$ but the final solution of eq 14 does not change for a nonreactive species.
} 
analyzed over time, either in the absence of any electric field (zero-field time-based experiments; Figure $\mathrm{S}-4 \mathrm{~b})$ or in the presence of an electric field $E_{y}$ of several $\mathrm{kVm}^{-1}$ oscillating at a few $\mathrm{Hz}$ along the $y$ axis ( $E_{y}$-field time-based experiments; Figure S-4c). The fluorescence intensity along $y$ averaged over a characteristic length $\Delta x(\sim 10 \mu \mathrm{m})$ is subsequently plotted as a function of time (Figures $\mathrm{S}-4 \mathrm{~b}$ and S-4b). The diffusion coefficient is eventually extracted from fitting experimental data with eq 16 after fast Fourier transform analysis.
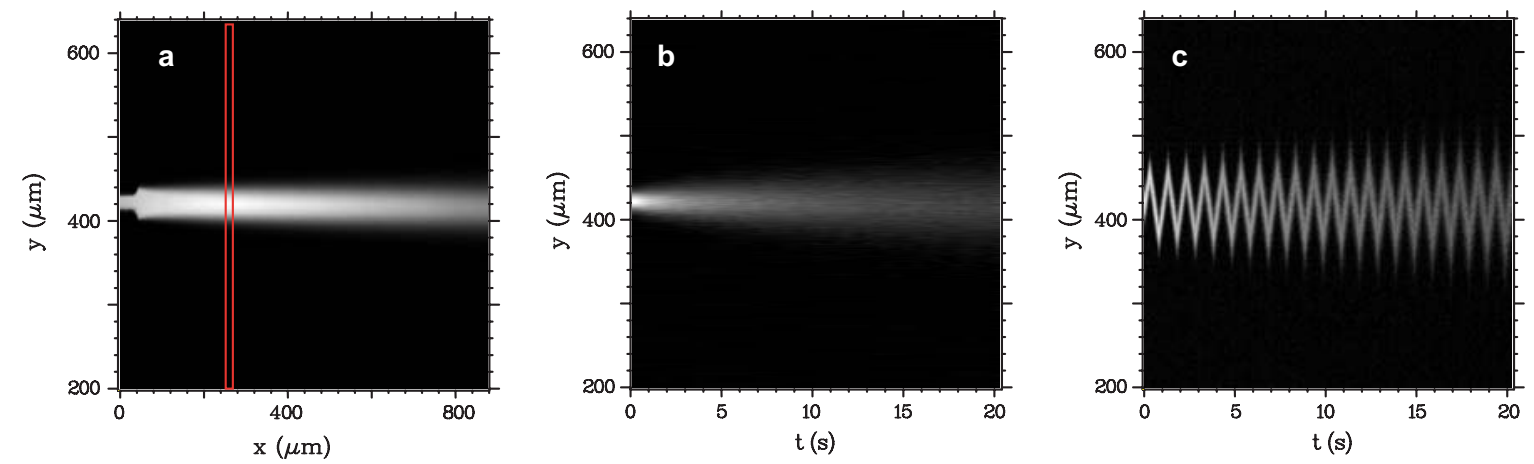

Figure S-4. Time-based experiments illustrated with $5 \mu \mathrm{M}$ YOYO-1 stained 151bp dsDNA (1:15 dye:bp ratio) in $\mathrm{NaOH} /$ Hepes $25 / 50 \mathrm{mM} \mathrm{pH} 7.5,0.1 \%$ (w/w) PDMA buffer at $20^{\circ} \mathrm{C}$. (a) Initial condition resulting from application of $E_{x}=8 \times 10^{3} \mathrm{Vm}^{-1}$. (b) Time-evolution of a free diffusing $y$-profile. To reduce noise, averaging is performed over $\Delta x=10$ pixels (red rectangle in the preceding pannel). (c) Time-evolution of the same $y$-profile when a $1 \mathrm{~Hz}$ alternative electric field $E_{y}=8 \times 10^{3} \mathrm{Vm}^{-1}$ is applied along the $y$-axis.

\section{Results and Discussion}

Figures S-4b and S-4c display the results for the weakly diffusing YOYO-1 stained $151 \mathrm{bp}$ dsDNA in zero-field and $E_{y}$-field time-based experiments. After 1D-Fourier transform analysis, we extracted the same $17 \pm 2 \times 10^{-12} \mathrm{~m}^{2} \mathrm{~s}^{-1}$ value of the diffusion coefficient from both measurements.

The $D_{0}$ diffusion coefficient obtained from the zero-field time-based experiment directly reflects Brownian motion: $D_{0}=D_{\text {Brown }}$. In contrast, additional phenomena might affect the corresponding value $D_{E_{y}}$ extracted from the $E_{y}$ time-based experiment. First, the different zeta-potentials of the lower and upper chamber walls may induce a linear dependence on $z$ of the fluid flow that gives rise to a Taylor contribution $D_{\text {Taylor }}$ to the diffusive behavior. Analyte adsorption and material heterogeneities could additionally generate variations of fluid velocity in the $x-y$ plane yielding a supplementary convective contribution to the diffusive relaxation: $D_{\text {convection. }}$. Thus two electric field-induced dispersions are expected to superimpose to the Brownian motion in the $E_{y}$-field time-based experiment: ${ }^{3}$

$$
D_{E_{y}}=D_{\text {Brown }}+D_{\text {Taylor }}+D_{\text {convection }}
$$

The fair agreement obtained here between the diffusion coefficients measured in Figure S- $4 b$ and S-4c suggests $\frac{D_{\text {Taylor }}+D_{\text {convection }}}{D_{\text {Brown }}} \ll 1$. Hence we conclude from this series of experiments that electroosmotic mobility is constant inside the analysis chamber. 


\section{Correction of the Optical Pattern in the Fourier Space}

\section{Exact Theoretical Computation}

At the present $\mathbf{F}$ concentrations, the molecular $\mathbf{F}$ brightness $Q_{F}$ is constant and inner filter effects are absent. ${ }^{2}$ Thus the fluorescence emission from the measurement chamber reflects the concentration $F(x, y)$ in analyte $\mathbf{F}$. However the amount of light collected on each camera pixel $I(x, y)$ depends also on the intensity of the excitation light at the device and on the photon collection efficiency for the CCD sensor area of the camera. We eventually write:

$$
I(x, y)=Q_{F} F(x, y) P(x, y)
$$

where we introduced $P(x, y)$ as an optical pattern function characterizing the set-up.

In the ideal case, $P(x, y)$ is constant over the focal plane. Then $I(x, y)$ and $F(x, y)$ exhibit a same functional dependence on $x$ and $y$ and the diffusion coefficient can be directly extracted from fitting the dependence of $\tilde{I}(x, q)$ on $x$ with eq 5 of the Main Text. In contrast, the present instrumental set-up favors a wide field of view together with a good level of sensitivity. The light source is correspondingly strongly focussed, which yields illumination inhomogeneities: $P(x, y)$ exhibits a 20 to $30 \%$ variation over the $\sim 1 \mathrm{~mm}$ field of view (Figure S-5a and S-5b). Further analysis is therefore required to extract the diffusion coefficient from the spatial dependence of $\tilde{I}(x, q)$.

Eq 19 shows that the discrete ${ }^{3}$ Fourier transform $\tilde{I}(x, n)$ is a convolution product between the corresponding terms $\tilde{F}(x, n)$ and $\tilde{P}(x, n)$ associated to the $\mathbf{F}$ concentration profile and to the optical pattern function respectively:

$$
\tilde{I}(x, n)=Q_{F} \sum_{k=-\infty}^{+\infty} \tilde{F}(x, n-k) \tilde{P}(x, k)
$$

or

$$
\tilde{I}(x, n)=Q_{F} \sum_{k=0}^{n} \tilde{F}(x, n-k) \tilde{P}(x, k)
$$

because $\tilde{F}(x, n)$ and $\tilde{P}(x, n)$ are only defined for $n \geq 0$. We eventually derive: $:^{4}$

$$
\frac{\tilde{I}(x, n)}{\tilde{I}(x, 0)}=\frac{\tilde{F}(x, n)}{\tilde{F}(x, 0)}+\sum_{k=1}^{n} \frac{\tilde{F}(x, n-k)}{\tilde{F}(x, 0)} \times \frac{\tilde{P}(x, k)}{\tilde{P}(x, 0)} .
$$

${ }^{3}$ We apply on the recorded $I(x, y)$ function a discrete Fourier transform defined as:

$$
\tilde{I}(x, n)=\frac{1}{\ell_{y}} \sum_{y=0}^{\ell_{y}-1} I(x, y) e^{-i q_{n} y}=\frac{1}{\ell_{y}} \sum_{y=0}^{\ell_{y}-1} I(x, y) e^{-2 \pi i n \frac{y}{\ell_{y}}}
$$

where $q_{n}=2 \pi n / \ell_{y}$ is the spatial frequency associated to the Fourier mode $n$ and $\ell_{y}$ the image size along $y$, in pixels.

${ }^{4}$ For a mixture, $I(x, y)$ results from the contributions of all the $\mathbf{F}_{j}$ analytes and eq 21 becomes:

$$
\frac{\tilde{I}(x, n)}{\tilde{I}(x, 0)}=\frac{\sum_{j} Q_{j} \tilde{F}_{j}(x, n)}{\sum_{j} Q_{j} \tilde{F}_{j}(x, 0)}+\sum_{k=1}^{n} \frac{\sum_{j} Q_{j} \tilde{F}_{j}(x, n-k)}{\sum_{j} Q_{j} \tilde{F}_{j}(x, 0)} \times \frac{\tilde{P}(x, k)}{\tilde{P}(x, 0)}
$$

for $n \geq 1$, yielding at zeroth order (vide infra):

$$
\frac{\tilde{I}(x, n)}{\tilde{I}(x, 0)}=\frac{\sum_{j} Q_{j} \tilde{F}_{j}(x, n)}{\sum_{j} Q_{j} \tilde{F}_{j}(x, 0)} .
$$



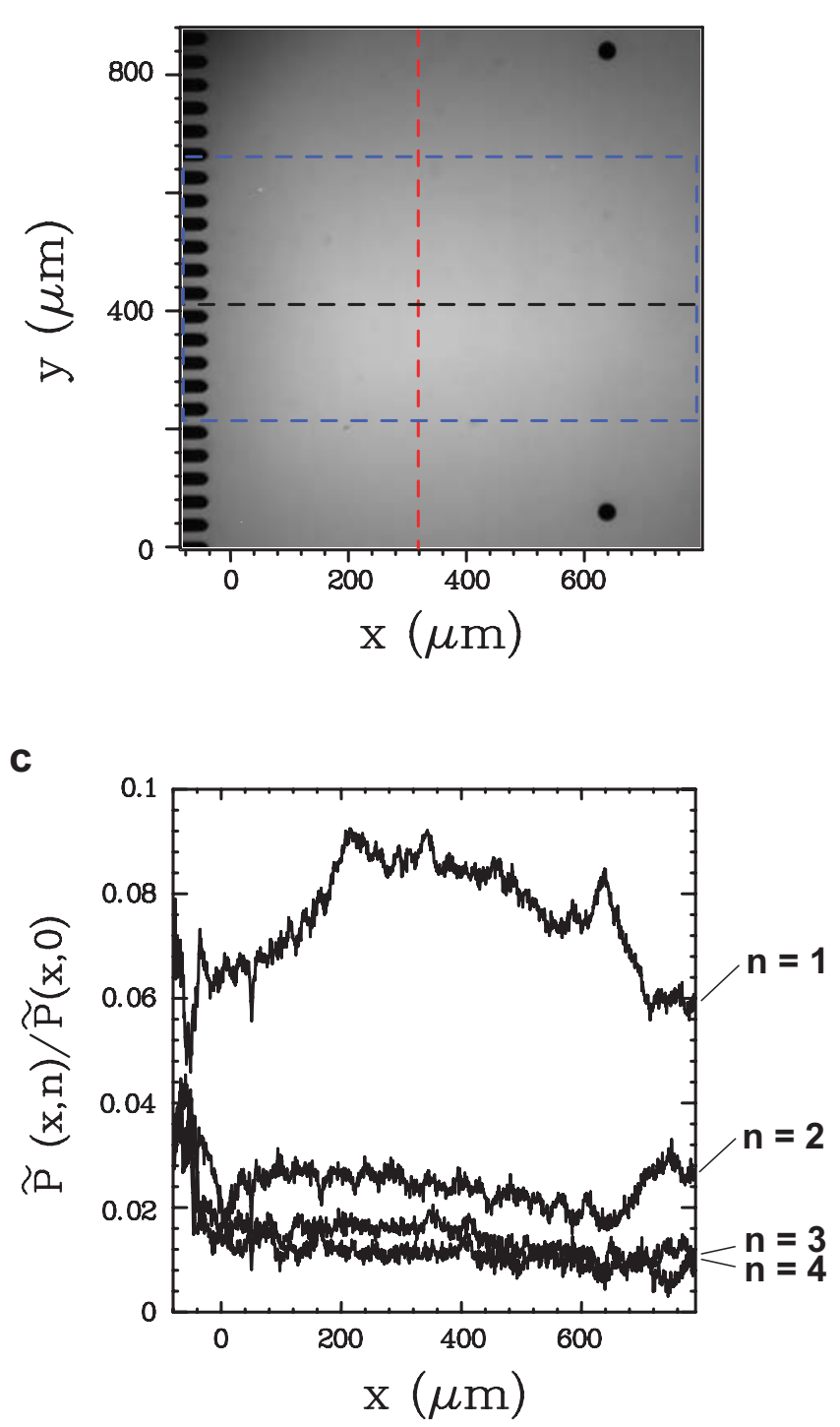

b

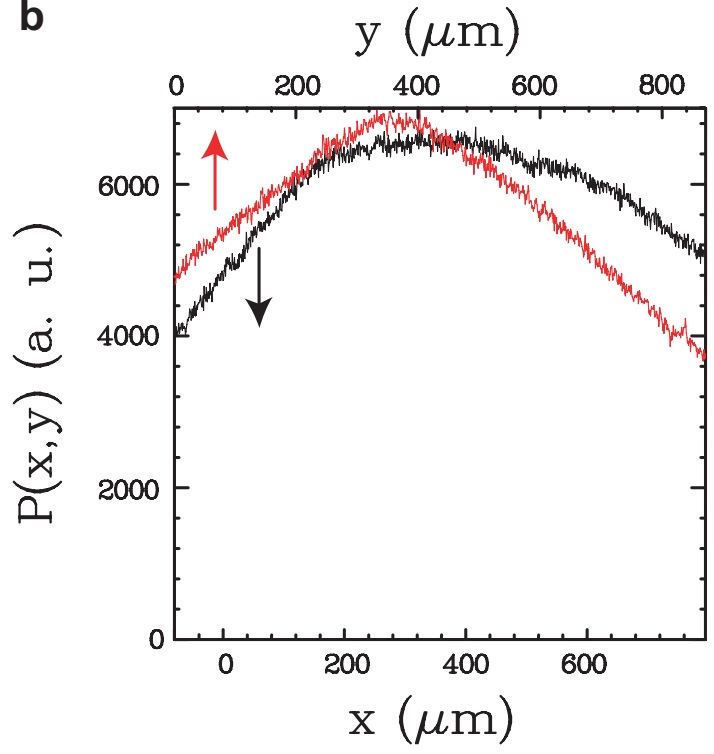

d
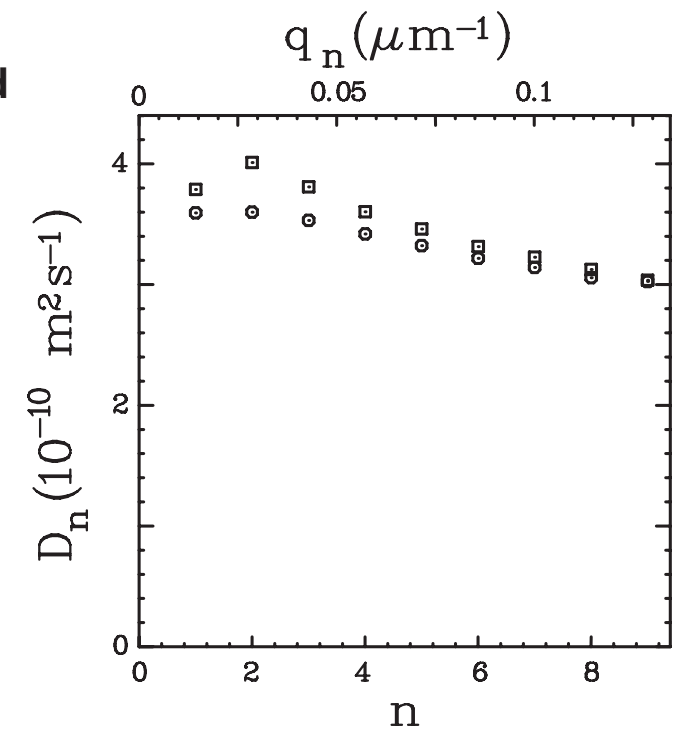

Figure S-5. Measurement of the illumination inhomogeneities and evaluation of the correction by zeroth Fourier mode normalization. (a) Epifluorescence image $P(x, y)$ of the analysis chamber homogeneously filled with $5 \mu \mathrm{M}$ fluorescein in $\mathrm{NaOH} / \mathrm{Hepes} 25 / 50 \mathrm{mM} \mathrm{pH} 7.5,0.1 \%(\mathrm{w} / \mathrm{w})$ PDMA at $20^{\circ} \mathrm{C}$. The blue dashed rectangle defines the area used for subsequent data treatment. (b) Intensity profiles along $x(-)$ and $y(-)$, respectively at the positions indicated by the dashed black and red lines in the preceding pannel. (c) Spatial dependence along $x$ of the $\tilde{P}(x, n) / \tilde{P}(x, 0)$ normalized Fourier modes resulting from the Fourier transform along $y$ of the chamber image ( $\ell_{y}=512$ pixels). (d) Dependence of the fluorescein diffusion coefficients on Fourier index $n$ (bottom scale) or on the spatial frequency $q_{n}$ (upper scale). Data were extracted from monoexponential fit of Figure $3 \mathrm{~b}$ of the Main Text, either with a zeroth $(O)$ or first $(\square ; \tilde{P}(x, 1) / \tilde{P}(x, 0)=0.08$ from pannel c) order correction (see eqs 22 and 23).

\section{Evaluation of the Zeroth Order Correction}

The second term in the right hand of the eq 21 complicates the extraction of the diffusion coefficient from the $\tilde{I}(x, n) / \tilde{I}(x, 0)$ spatial dependence. Indeed, the simple exponential dependence given in eq 5 of the Main Text only applies for $\tilde{F}(x, n)$. In the following, we measure $\tilde{P}(x, n) / \tilde{P}(x, 0)$ to evaluate at 
which order $\frac{\tilde{I}(x, n)}{\tilde{I}(x, 0)}$ has to be corrected to facilitate analysis without introducing any detrimental error.

Figures S-5a and S-5b display the epifluorescence image $P(x, y)$ of the analysis chamber homogeneously filled with $5 \mu \mathrm{M}$ fluorescein in $\mathrm{NaOH} /$ Hepes $25 / 50 \mathrm{mM}$ pH 7.5. Figure $\mathrm{S}-5 \mathrm{c}$ shows the corresponding spatial dependence along $x$ of the $\tilde{P}(x, n) / \tilde{P}(x, 0)$ normalized Fourier modes resulting from the Fourier transform along $y$. The $\tilde{P}(x, n) / \tilde{P}(x, 0)$ terms rapidly decrease from 0.08 for $n=1$ to nearly 0.01 for $n \geq 2$.

The latter observation suggests to analyze our images at the zeroth order by identifying:

$$
\frac{\tilde{F}(x, n)}{\tilde{F}(x, 0)}=\frac{\tilde{I}(x, n)}{\tilde{I}(x, 0)}
$$

However, to evaluate the corresponding level of approximation, we also computed first-order corrected concentration functions as:

$$
\frac{\tilde{F}(x, n)}{\tilde{F}(x, 0)}=\frac{\tilde{I}(x, n)}{\tilde{I}(x, 0)}-\frac{\tilde{F}(x, n-1)}{\tilde{F}(x, 0)} \times \frac{\tilde{P}(x, 1)}{\tilde{P}(x, 0)}
$$

Figure S-5d displays for both corrections the diffusion coefficients extracted from analysis of the data shown in Figure 4b. The agreement between both sets of values improves with the mode index $n$ and is always better than our 10\% estimation for the experimental errors (Table 1 and 2).

In the present work, we correspondingly retained the simplest zeroth Fourier mode normalization for data correction and each normalized Fourier mode $\tilde{I}(x, n) / \tilde{I}(x, 0)$ was fitted with the $\frac{\tilde{F}(0, n)}{\tilde{F}(0,0)} \exp \left(\frac{1-\sqrt{1+4 D^{2} q^{2} / v_{x}^{2}}}{2 D / v_{x}} x\right)$ function. 


\section{Validation of the procedure for data processing}

To evaluate our procedure for data processing, we generated the migration-diffusion concentration pattern of fluorescein by Finite Element Method (FEM) simulations and analyzed it by Fourier transform. With regard to the experiment, such a simulation deals with an ideal behavior and does not depend on the illumination pattern. Figure $3 \mathrm{c}$ of the Main Text compares the experimental $D_{n}$ values with the ones extracted from the FEM simulations. A fair agreement is observed which underlines in particular the relevance of the zeroth order correction for illumination inhomogeneities which assimilates $\tilde{I}(x, n) / \tilde{I}(x, 0)$ to $\tilde{F}(x, n) / \tilde{F}(x, 0)$ for data processing.

We also took advantage of the Gaussian shape of the profile of fluorescence emission at $x=0$ (see Figure S-8e) to evaluate the relevance of the Fourier analysis to extract diffusion coefficients from experimental data. Results extracted either from Fourier transform or from Gaussian fitting, when the latter can be applied i.e. in the 1D limit, were in agreement within experimental error (see Table S-3). 


\section{Supplementary Tables}

Table S-2. Sequences ( $5^{\prime}-3$ ' orientation) of the rhodamine-green (rhg) and texas-red (tex) labelled singlestranded oligonucleotides used in this study. The complementary sequence of C100 to tex-9 is higlighted in bold, mismatches are underlined.

\begin{tabular}{|c|c|}
\hline Name & Sequence \\
\hline rhg-13 & rhg-TCCTTTGTTTGTG \\
\hline rhg-27 & rhg-TAGTATTATCTTACACAAACAAAGGAG \\
\hline \multirow[t]{3}{*}{ rhg-105 } & rhg-TAGTATTATCTTACACAAACAAAGGAGAATAAAATGAAAAC \\
\hline & -GATTAATCTGAACGCTGCAGTTAAAACTAAATGCTTCAATGGT \\
\hline & -AAATATGATGAAACTATGTGG \\
\hline tex-9 & tex-CTTTGTTTG \\
\hline tex $-9 \mathrm{~m}$ & tex-ATTTGTTTG \\
\hline tex $-9 \mathrm{M}$ & 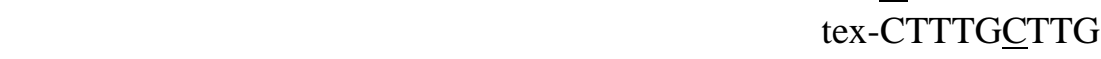 \\
\hline \multirow[t]{3}{*}{$\mathrm{C} 100$} & TAGTATTATCTTACACAAACAAAGGAGAATAAAATGAAAAC \\
\hline & -GATTAATCTGAACGCTGCAGTTAAAACTAAATGCTTCAATGGT \\
\hline & -AAATATGATGAAACTA \\
\hline
\end{tabular}


Table S-3. Averaged diffusion coefficients $\langle D\rangle\left(10^{-12} \mathrm{~m}^{2} \mathrm{~s}^{-1}\right)$ for fluorescein, rhg-13 - a 13 bases long rhodamine-green labelled oligonucleotide, and YOYO-1 stained dsDNA of different lengths (1:15 dye:bp ratio) in $\mathrm{NaOH} / \mathrm{Hepes} 25 / 50 \mathrm{mM} \mathrm{pH} \mathrm{7.5,} 0.1 \%$ (w/w) PDMA buffer at $20^{\circ} \mathrm{C}$. Measurements were performed in zero-field time-based experiments and were analyzed by either Fast Fourier Transform or Gaussian fitting in the direct space. Errors correspond to standard deviation.

\begin{tabular}{lrrrrr}
\hline Analysis method & Fluorescein & rhg-13 & 151bp & 438bp & $1257 \mathrm{bp}$ \\
\hline FFT & $330 \pm 10$ & $106 \pm 5$ & $17 \pm 2$ & $8.6 \pm 0.8$ & $4.2 \pm 0.3$ \\
Gaussian & $330 \pm 30$ & $103 \pm 5$ & $19 \pm 2$ & $9.2 \pm 0.4$ & $4.4 \pm 0.4$ \\
\hline
\end{tabular}




\section{Supplementary Figures}

a

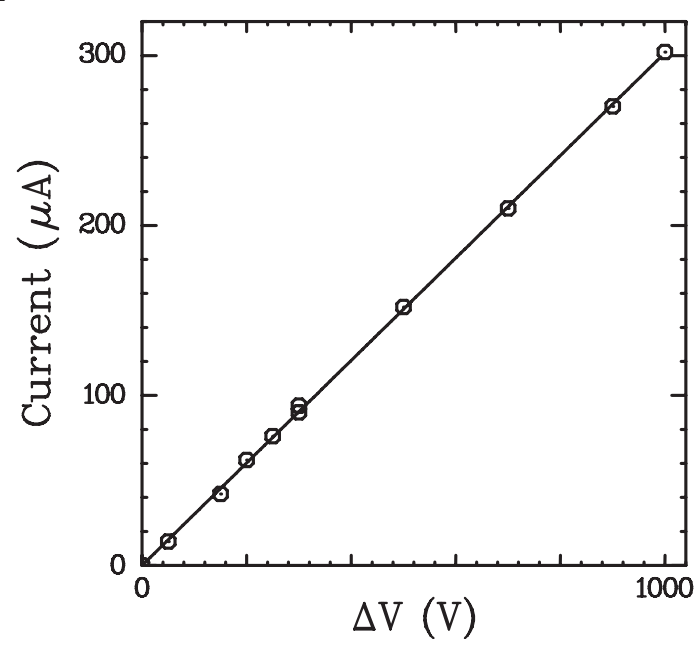

b

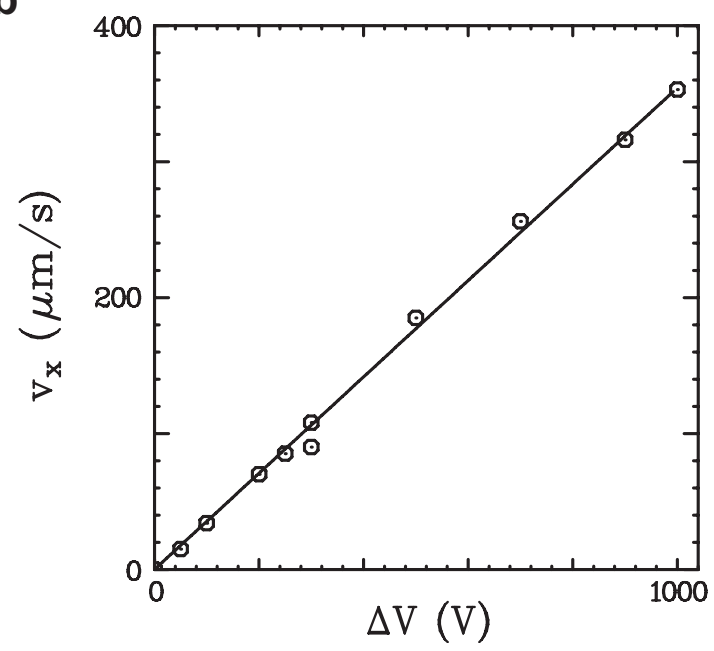

Figure S-6. Electric current (a) and migration velocity for fluorescein (b) as a function of the voltage drop applied between the two reservoirs located along the $x$ axis. The microscope stage was thermostated at $20^{\circ} \mathrm{C}$ and the chip was filled with $\mathrm{NaOH} / \mathrm{Hepes} 25 / 50 \mathrm{mM} \mathrm{pH} \mathrm{7.5,1 \% (w/w)} \mathrm{agarose} \mathrm{medium.} \mathrm{The} \mathrm{linearity} \mathrm{observed} \mathrm{in} \mathrm{both} \mathrm{figures}$ underlines the absence of any departure from the preset temperature: Heat produced by Joule effect is efficiently dissipated even at the highest applied voltages. 


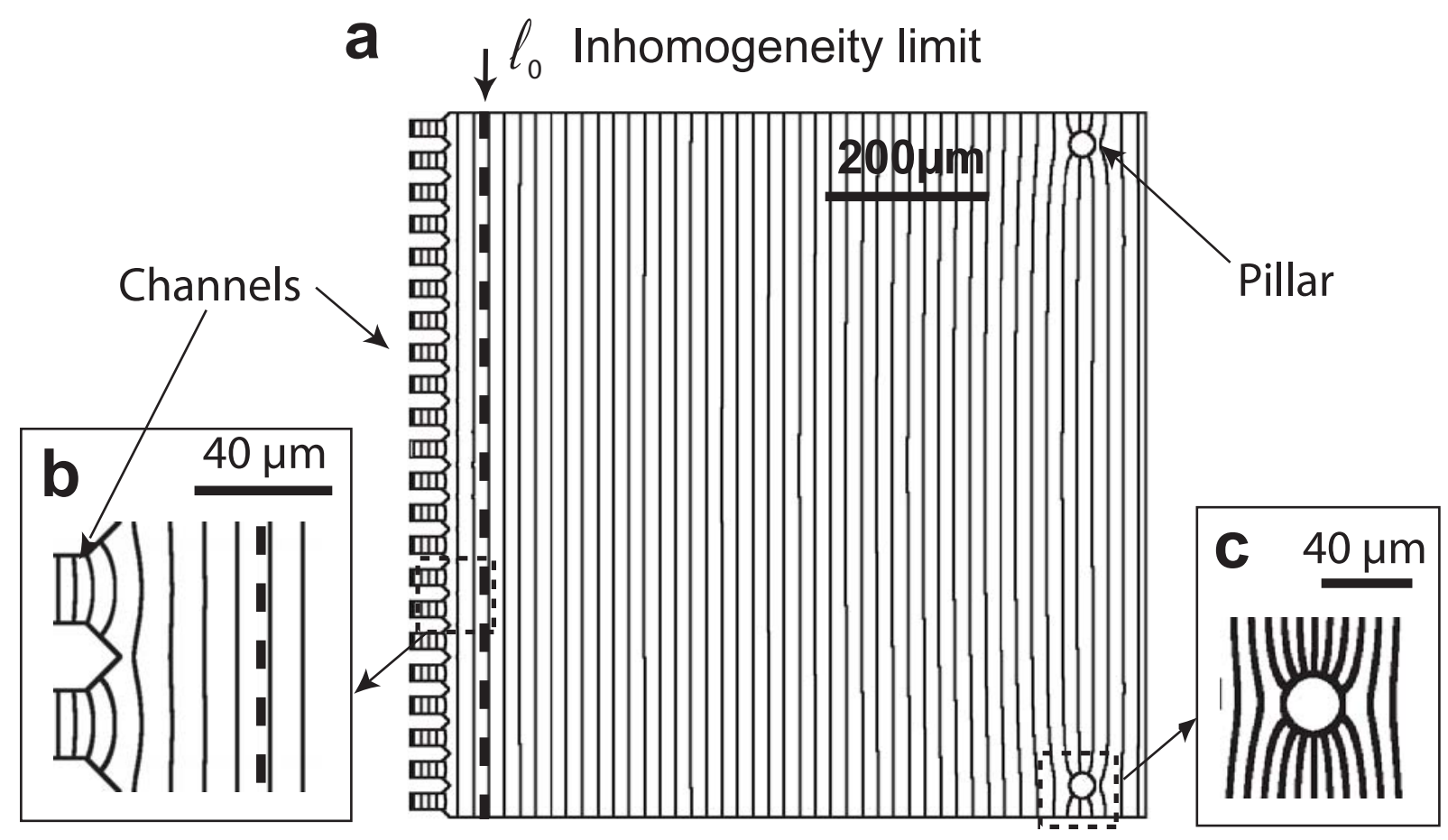

Figure S-7. Isopotentials of the electric field calculated by Finite Element Method simulations. (a) Area corresponding to the camera field of view. Each line accounts for a $2 \%$ variation. (b) and (c) Respective zooms at the boundary between the channels and the chamber and around a pillar. Each line corresponds to a $1 \%$ variation. After a distance $\ell_{0}=40 \mu \mathrm{m}$ (dashed line), the electric field is essentially homogeneous and parallel to the $x$-axis. 

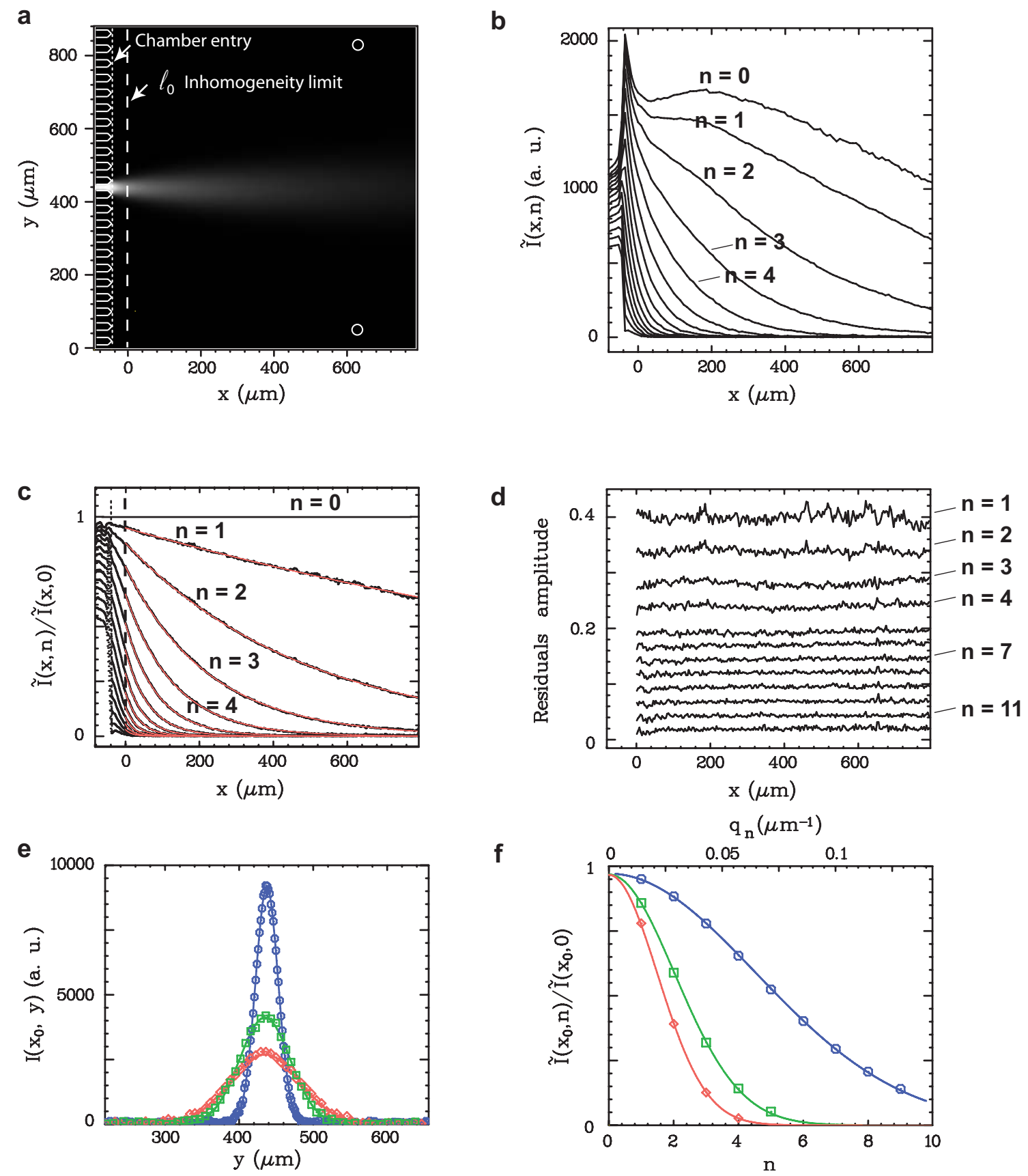

Figure S-8. Detailed data treatment for the diffusion coefficient measurement by 1D-Fourier transform analysis. (a) Stationary migration-diffusion pattern obtained for $5 \mu \mathrm{M}$ fluorescein when an electric field of $8 \times 10^{3} \mathrm{Vm}^{-1}$ $\left(v_{x}=145 \mu \mathrm{ms}^{-1}\right.$ ) is applied left to right in NaOH/Hepes $25 / 50 \mathrm{mM} \mathrm{pH} \mathrm{7.5,0.1 \% (w/w)} \mathrm{PDMA} \mathrm{buffer} \mathrm{at} 20^{\circ} \mathrm{C}$. (b) Spatial dependence along $x$ of the Fourier modes resulting from the Fourier transform of the preceding image along $y$. (c) Fourier mode normalization by the zeroth Fourier mode. The truncated $\tilde{I}(x, n) / \tilde{I}(x, 0)$ data are fitted by monoexponentials $(-)$. (d) Corresponding residuals. To improve readability, a vertical offset was added. (e) Intensity profiles along $y$ at $x_{0}=0(0), 200(\square)$, and $400 \mu \mathrm{m}(\diamond)$. All the three data sets are satisfactorily fitted by a Gaussian function. (f) Corresponding normalized Fourier amplitudes plotted as a function of the Fourier index $n$. Gaussian fits are again appropriate. 
a

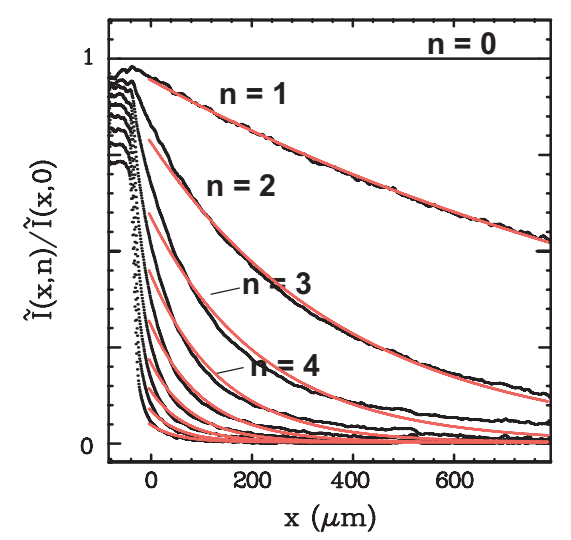

C

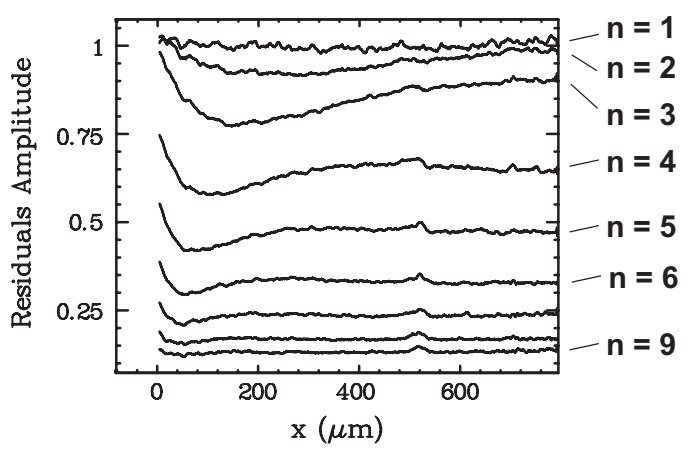

b

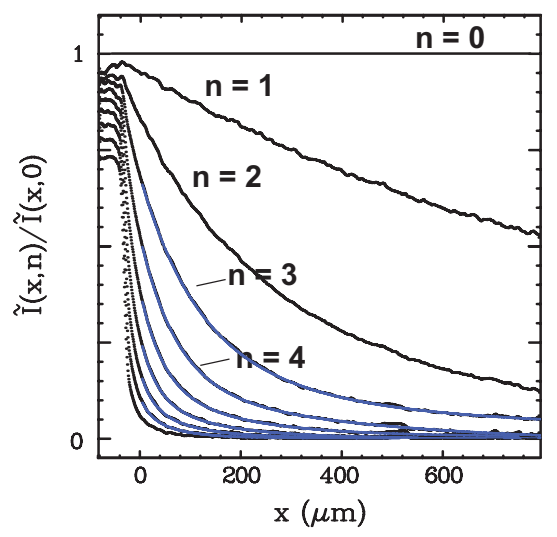

d

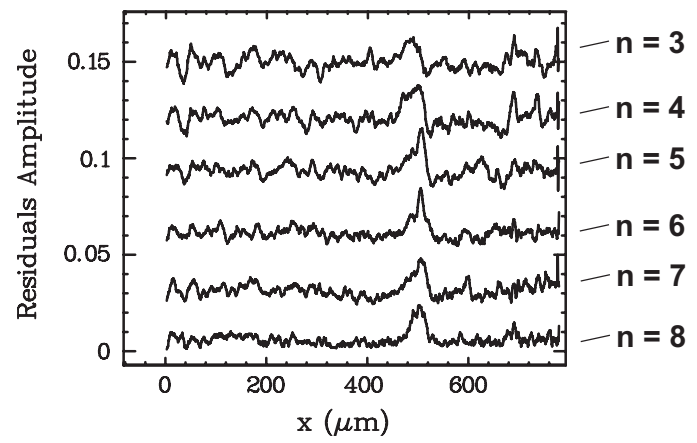

Figure S-9. Comparison of the mono and biexponential fitting procedures for analysis of a binary mixture. The stationary migration-diffusion pattern was obtained by applying an electric field $E_{x}=266 \mathrm{Vm}^{-1}\left(v_{x}=\right.$ $4.8 \mu \mathrm{ms}^{-1}$ ) left to right in $\mathrm{NaOH} /$ Hepes $25 / 50 \mathrm{mM} \mathrm{pH} \mathrm{7.5,0.1 \% (w/w)} \mathrm{PDMA} \mathrm{buffer} \mathrm{at} 20^{\circ} \mathrm{C}$. The binary mixture was composed of two dsDNA, 151 and 1257bp long stained with YOYO-1 (1:15 dye:bp). They were in relative abundance $\alpha(151 b p / 1257 b p)=2.2$. (a) Monoexponential and (b) biexponential fits of the spatial dependence in $x$ of the normalized Fourier modes. (c) and (d) residuals corresponding to the mono and biexponential fits, respectively. To improve readability, a vertical offset was added. The residuals shown in Figure S-9c clearly contradict the diffusive behavior of a single component. 

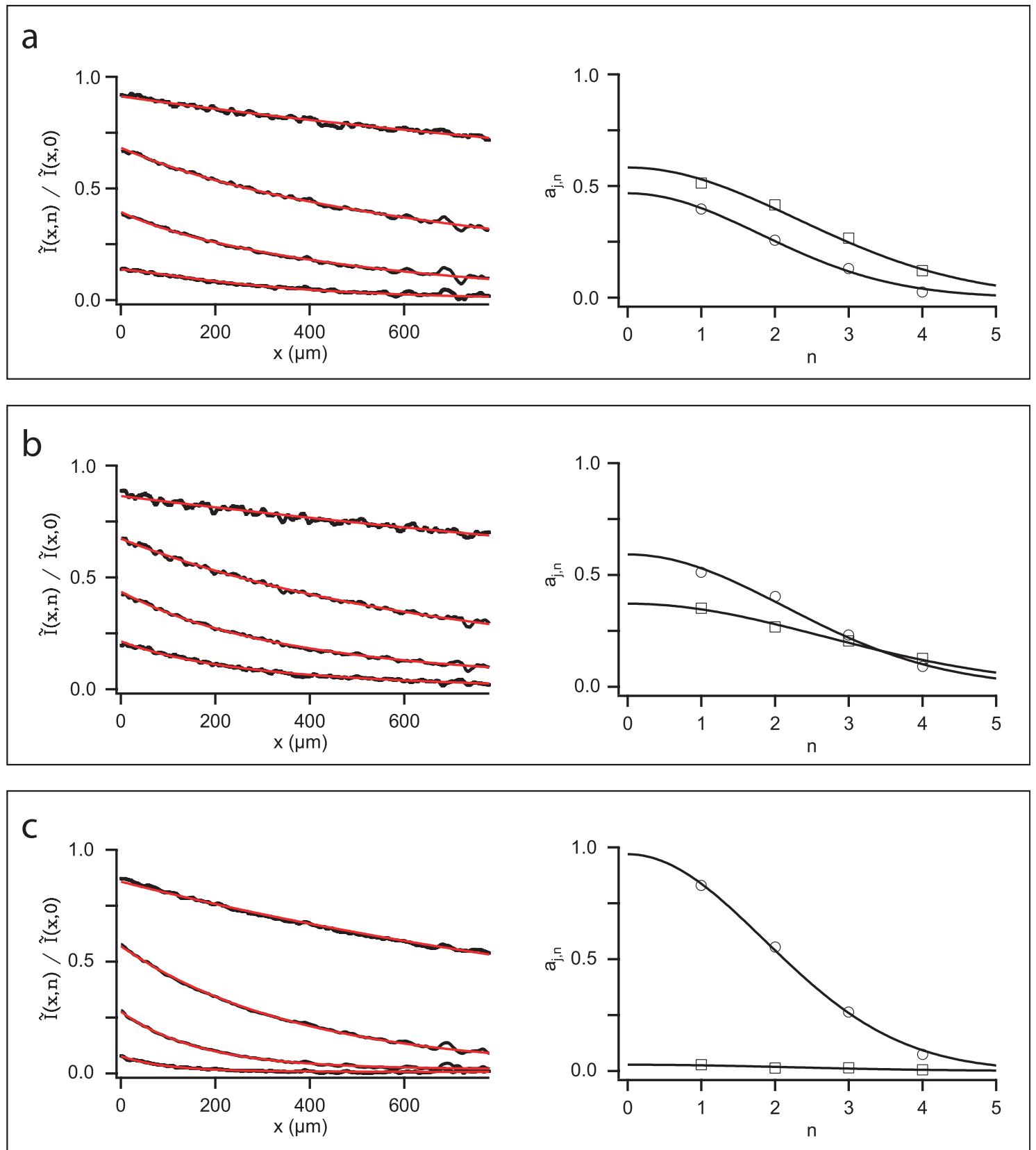

Figure S-10. Affinity screening of three texas red-labelled 9mer olignucleotides at $1 \mu \mathrm{M}$ for a target oligonucleotide $\mathrm{C} 100$ at $5 \mu \mathrm{M}$ in $\mathrm{NaOH} / \mathrm{Hepes} 25 / 50 \mathrm{mM} \mathrm{pH} 7.5,0.1 \%$ (w/w) PDMA buffer at $10^{\circ} \mathrm{C}$ : (a) tex-9+C100, (b) tex $-9_{m}+\mathrm{C} 100$ and (c) tex $-9_{M}+C 100$. Left plots display the relaxation along $x$ of the normalized Fourier modes (experimental data: black lines; red lines: biexponential fits). Right plots show the amplitudes of the biexponential fits for the duplex $(\square)$ and the free probe $(O)$ as a function of the Fourier mode $n$. Solid lines correspond to gaussian fits. The relative abundances $\alpha_{\text {exp }}$ were calculated as $a_{d u p l e x, 0} / a_{\text {free probe, } 0}$. In the case of tex- $9_{\mathrm{M}}$, essentially no double stranded species is formed and the application of the biexponential analysis yields a zero diffusion coefficient. 


\section{References}

[1] http://frodo.wi.mit.edu/cgi-bin/primer3/primer3.cgi/

[2] Valeur, B. Molecular Fluorescence; Wiley-VCH: Weinheim; 2002.

[3] Giddings J. C. Unified Separation Science; Wiley: New York; 1991. 